\title{
Fructus mume extracts alleviate cognitive impairments in 5XFAD transgenic mice
}

\author{
Jung-Cheol Park', Jinhua Ma', Won Kyung Jeon ${ }^{2^{*}}$ and Jung-Soo Han ${ }^{1^{*}}$
}

\begin{abstract}
Background: Fructus mume (F. mume) has been used as a traditional treatment for ulcer, cough, and digestive problems for many years in Asian countries. Previous studies have demonstrated that $F$. mume extracts alleviate cognitive deficits in rats with chronic cerebral hypoperfusion and in mice with scopolamine treatments. The present experiment was conducted to examine the effects of $F$. mume on cognitive impairments in SXFAD transgenic mice with five familial Alzheimer's disease (AD) mutations.

Methods: F. mume was administered daily to 5XFAD mice at 12 weeks of age and continued for 90 days. Cognitive function was evaluated using a spatial memory version of the Morris water maze task, the object/location novelty recognition test, and contextual fear conditioning at 24 weeks of age. To elucidate the possible mechanisms underlying the memory improving effects of F. mume in 5XFAD mice, we examined alterations in hippocampal cholinergic function.

Results: Vehicle-treated 5XFAD mice exhibited hippocampus-dependent memory impairments compared with non-transgenic littermates, which was reversed in F. mume-treated 5XFAD mice. In addition, reduced hippocampal choline acetyltransferase (ChAT) levels in 5XFAD mice were reversed by F. mume treatment, indicating that F. mume enhances the effects of cholinergic neuronal function.
\end{abstract}

Conclusions: F. mume may have therapeutic effects on cognitive impairments in AD.

Keywords: Fructus mume, Alzheimer's disease, Cognition, Choline acetyltransferase, 5XFAD

\section{Background}

Fructus mume (F. mume), the processed unripe fruit of Prunus mume, has been used as a traditional treatment for many years in Asian countries. F. mume has been reported to be effective in a rat model of colitis induced by 2,4,6-trinitrobenzene sulfonic acid [1], and is inhibitory to pro-inflammatory mediators in lipopolysaccharide (LPS)-stimulated RAW 264.7 cells [2]. We recently reported that $F$. mume treatment can alleviate impaired cognitive function in a rat model of vascular dementia $(\mathrm{VaD})$ induced by a bilateral common carotid artery occlusion (BCCAo) [3-6]. We also examined the effects of F. mume on scopolamine-induced cognitive impairment in mice [7]. In agreement with the results of the BCCAo

\footnotetext{
* Correspondence: wkjeon@kiom.re.kr; jshan06@konkuk.ac.kr

${ }^{2}$ Herbal Medicine Research Division, Korea Institute of Oriental Medicine, Daejeon 305-811, Republic of Korea

'Department of Biological Sciences, Konkuk University, 1 Hwayang-dong, Gwangjin-gu, Seoul 143-701, Republic of Korea
}

study, treatment with $F$. mume improved cognitive impairments in scopolamine-injected mice [7].

These latter two studies $[6,7]$ also found that $F$. mume has anti-inflammatory effects and enhances central cholinergic function. Based on this reported action of $F$. mume, the present experiment was conducted to examine the therapeutic potential of F. mume for Alzheimer's disease (AD), using an animal AD model.

$\mathrm{AD}$ is characterized by a progressive loss of cognitive function. The neuropathological hallmarks are the accumulation of extracellular senile plaques and intraneuronal fibrillary tangles, as well as the loss of basal forebrain cholinergic neurons $[8,9]$. In particular, numerous studies indicate that basal forebrain cholinergic neurons, which provide the major innervation of the hippocampus and cortex, play an important role in cognitive function [10-12]. The loss of basal forebrain cholinergic neurons has been reported in the brains of patients with $\mathrm{VaD}$ and $\mathrm{AD}$. In addition, neurodegeneration of basal 
forebrain cholinergic neurons has been observed in the brains of BCCAo rats and AD transgenic mice [13-17]. Therefore, given that F. mume ameliorated BCCAoinduced and scopolamine-induced cognitive impairments, we expected beneficial effects of $F$. mume on cognitive deficits in AD.

To investigate whether F. mume mitigates cognitive impairments in $\mathrm{AD}$, we used 5XFAD transgenic mice cooverexpressing human amyloid precursor protein (APP) and presenilin-1 (PS1) with five familial AD (FAD) mutations. 5XFAD mice generate and accumulate cerebral amyloid- $\beta$ (A $\beta) 42$ and begin to develop visible amyloid deposits at 2 months of age [18]. 5XFAD mice exhibit memory decline, especially in hippocampus-dependent behavioral tasks, at 4-6 months of age [19, 20]. In addition, 5XFAD mice exhibit reduced choline acetyltransferase (ChAT) levels in the hippocampus and loss of cholinergic neurons in the medial septum (MS) and the vertical limb of diagonal band (VDB) at 6 months of age [21]. In the present study, 5XFAD mice were administered F. mume for approximately 3 months and then followed several behavioral tasks for assessing cognitive function. F. mume treatment alleviated cognitive impairments via increases in hippocampal ChAT levels.

\section{Methods}

\section{Preparation of $F$. mume extracts}

F. mume was purchased from a commercial supplier (Kwangmyung-Dang, Ulsan, Korea) in 2010. It was identified by the Herbal Quality Control Team and deposited at the Creative Research Laboratory, KIOM (Korea). Dried F. mume was pulverized and extracted with distilled water $(2 \mathrm{~kg} / 8 \mathrm{~L})$ for $2 \mathrm{~h}$ below $100{ }^{\circ} \mathrm{C}$ in an ultrasound-assisted extractor (OM30-EP; Sonimedi, Korea). All extracts were concentrated under vacuum using a rotary evaporator after filtration and were then dried for $48 \mathrm{~h}$ at $40{ }^{\circ} \mathrm{C}$ by using an extract vacuum drier (Exdryer, Sonimedi, Korea) to yield a powder extract (324.5 g, $16.225 \%$ yield). The powder extract was suspended in sterilized distilled water at the appropriate concentrations. An HPLC assay was performed with citric acid as a standard maker for quality control of the $F$. mume extract composition in each experiment. HPLC was performed using two Waters 515 pumps, a 2996 photodiode array detector, and a Phenomenex Synergi Hydro RP-80A (4 m, 4.6 × $250 \mathrm{~mm}$ i.d.). The mobile phase was composed of acetonitrile (A) and $0.1 \%$ phosphoric acid (B) with a linear gradient elution: 0 min, $100 \%$ B; 15 min, 3.8 \% A. F. mume extract was filtered on membrane filters with a $0.45 \mathrm{~mm}$ pore size (Millipore) and a $10 \mathrm{~L}$ injection volume. Citric acid was detected at a wavelength of $220 \mathrm{~nm}$. The crude extract was analyzed in triplicate, and the citric acid content was found to be about $18.68 \%[6,22]$.

\section{Animals and $F$. mume extract administration}

The generation of 5XFAD mice (Tg6799 line) has been described previously [18]. These mice co-express and co-inherit both mutant human APP (695) with the Swedish (K670N, M671L), Florida (I716V), and London (V717I; FAD) mutations and human PS1 harboring two FAD mutations, M146L and L286V. The expression of these transgenes is regulated by neuron-specific mouse Thy-1 promoters to drive overexpression in the neurons. The 5XFAD strain (B6/SJL genetic background) was maintained by crossing hemizygous transgenic mice with non-transgenic B6/SJL breeders, and 5XFAD transgenic mice were used for the experiments with non-transgenic littermates as controls. Before the experiments, genomic DNA was extracted from the tail tips of all mice and genotyping was performed by polymerase chain reaction analysis. 23 non-transgenic controls (18 male, 5 female) and 38 5XFAD ( 28 male, 10 female) mice were used for the experiments. All mice had access to food and water ad libitum and were housed under a 12-12 h light-dark cycle at $22-24{ }^{\circ} \mathrm{C}$. All behavioral experiments were performed during the light phase.

Preparation of $F$. mume has been described previously [6]. Mice were administered F. mume extract $(200 \mathrm{mg} /$ $\mathrm{kg}$ ) or distilled water as a vehicle control by oral gavage. Treatment began when the mice were 12 weeks of age and continued until they were sacrificed, for approximately 3 months. The Institutional Animal Care and Use Committee of Konkuk University approved all protocols described in this report.

\section{Morris water maze task}

The Morris water maze test pool was $1.83 \mathrm{~m}$ in diameter, and $0.58 \mathrm{~m}$ in height, with a $0.2 \mathrm{~m}$ in diameter hidden platform and was surrounded by a white curtain marked with extra-visual cues. The pool was filled with $24-27{ }^{\circ} \mathrm{C}$ opaque water. Mice were trained on four trials per day for 8 consecutive days. Mice were allowed to search for the platform for $60 \mathrm{~s}$; if they did not find the platform, they were placed on the platform manually, where they were held for $15 \mathrm{~s}$. After the trials were completed, the mice were dried and placed back into their home cages. To assess memory retention, a probe test was performed on training day 5, 7, and 9. During the probe test, platform was removed and mice were allowed to swim for $30 \mathrm{~s}$. In all trials, the movements of the mice were recorded and analyzed using a HVS image tracking system (Hampton, UK).

\section{Contextual fear conditioning task}

Contextual fear conditioning task was conducted in square $(18 \mathrm{~cm} \mathrm{~W} \mathrm{x} 18 \mathrm{~cm} \mathrm{D} \mathrm{x} 30 \mathrm{~cm} \mathrm{H})$ chambers equipped with a steel-grid floor through which footshock could be delivered (Coulbourn, PA, USA). The 
task consisted of habituation, training, and test sessions. During habituation, the mice were placed in a conditioning chamber and allowed to explore without a foot shock for $12 \mathrm{~min}$. On the next day, during training, mice were placed in the conditioning chamber for $148 \mathrm{~s}$, and then received three unsignaled foot shocks $(0.75 \mathrm{~mA}$, $2 \mathrm{~s})$ at 30-s intervals. The test session was scheduled $24 \mathrm{~h}$ after training. During the test, mice were placed into the same conditioning chamber, and contextual fear memory was evaluated by scoring freezing behavior for $3 \mathrm{~min}$ by an investigator blind to condition. In addition, the baseline freezing behavior was analyzed during the 2 min before the delivery of the foot shock during the training session.

\section{Object/location novelty recognition test}

The object/location novelty recognition test consisted of four sessions: habituation, sample object phase, novel object phase, and novel object location phase. Each mouse was individually habituated to the open field box $(50 \times 50 \mathrm{~cm})$ for $15 \mathrm{~min}$. On the next day during the sample object phase, two identical sample objects were placed in the two corners of the box, and the mice were allowed to freely explore the arena and objects for $10 \mathrm{~min}$. The novel object phase was scheduled $24 \mathrm{~h}$ later, in which the mice were placed into the arena again, and one of the sample objects was replaced with a novel object. Mice were allowed to explore the arena for $5 \mathrm{~min}$, and the time spent exploring each object was scored for 2 min. $24 \mathrm{~h}$ later, during the novel object location phase, one of the two objects that were used in novel object phase was moved to a novel place in the arena, and the mice were tested in the same way as before. The discrimination ratio was calculated as the ratio of time spent exploring the novel object or the object moved to novel location to the total time spent exploring the two objects.

\section{Western blot analysis}

One week after the behavioral experiments, 9 mice per group were decapitated and the hippocampal or cortical samples were dissected and snap-frozen. For the total protein extracts, individual tissue samples were homogenized in ice-cold buffer containing $20 \mathrm{mM}$ Tris ( $\mathrm{pH} 7.5$ ), $5 \%$ glycerol, $1.5 \mathrm{mM}$ EDTA, $40 \mathrm{mM} \mathrm{KCl}, 0.5 \mathrm{mM}$ dithiothreitol, and protease inhibitors. The homogenates were then centrifuged at $14,000 \mathrm{rpm}$ for $1 \mathrm{~h}$ at $4{ }^{\circ} \mathrm{C}$, and the supernatant was harvested and stored at $-80^{\circ} \mathrm{C}$. The concentration of the protein extracts was determined by the Bradford assay. The protein extracts were separated by SDS-PAGE and transferred to a PVDF membrane using the Mini Trans-Blot Cell (Bio-Rad). After blocking, the membranes were incubated with anti-ChAT antibody (goat polyclonal; millipore, 1:1000), anti- $\beta$-actin antibody (sigma, 1:5000), and then with HRP-conjugated secondary antibodies. The signals were visualized by an ECL system and developed onto hyperfilm. The relative ChAT expression level was determined by densitometry using the Image Gauge software (Fujifilm) and was normalized to $\beta$-actin levels.

\section{Immunohistochemistry}

One week after the behavioral experiments, 6 mice per group were euthanized by ketamine and xylazine and were intracardially perfused with $4 \%$ paraformaldehyde (PFA) in phosphate buffered saline (PBS). Following fixation, the brains were removed to $4 \%$ PFA and $30 \%$ sucrose solutions, respectively, in order. Free-floating brain sections $(40 \mu \mathrm{m})$ were obtained from frozen-section mice brains using microtome (Leica). Endogenous peroxidase activity in the brain sections was quenched by incubation in $3 \% \mathrm{H}_{2} \mathrm{O}_{2} / 10 \% \mathrm{MeOH}$ in PBS. The sections were then incubated for $1 \mathrm{~h}$ at room temperature in PBS with $0.3 \%$ Triton-X 100 containing $10 \%$ fetal horse serum (GIBCO). The sections were incubated with anti-ChAT antibody (goat polyclonal; Millipore, 1:1000) overnight and then incubated for $1 \mathrm{~h}$ with the appropriate biotinylated secondary antibodies (Vector, 1:500) and for $2 \mathrm{~h}$ in extravidin peroxidase conjugate (Sigma Aldrich, 1:1000). Finally, the sections were reacted with a 3,3 -diaminobenzidine (DAB) substrate kit (Vector) and mounted onto resin coated slides. After dehydration in a series of ethanol and clearance in xylene, the slides were covered with a coverslip using permount reagent. To quantify the number of ChAT-positive cells, 8 sections from the medial septum region (between +1.2 and $+0.8 \mathrm{~mm}$ anterior to the bregma) and 4 sections from the substantia innominata region (between +0.3 and $+0.1 \mathrm{~mm}$ anterior to the bregma) were selected from each individual. To capture the images, light microscopy was conducted on ECLIPSE Ni-U microscope (Nikon, Japan) equipped with a ProgRes CFscan camera (JENOPTIK, Germany). After the same threshold level and region of interest were applied to each image, the number of ChAT-positive cells was counted using MetaVue software.

\section{Statistical analysis}

One-way analysis of variance (ANOVA) or two-way ANOVA with repeated measurements was conducted to assess the effects of the $F$. mume extract on the changes in hippocampal and cortical ChAT levels and the cognitive impairments in 5XFAD mice. Post-hoc analyses (Least Significant Difference test) were subsequently performed to determine the effects of the F. mume treatment. $P$ values of less than 0.05 were considered significant, unless otherwise specified. The data were 
expressed as the mean \pm standard error of the mean (SEM).

\section{Results}

F. mume alleviated cognitive impairments in 5XFAD mice

To examine the effect of $F$. mume on cognitive impairments in 5XFAD mice, several hippocampus-dependent behavioral tasks were conducted (Fig. 1). We first conducted the Morris water maze task to assess spatial memory. It has previously been reported that 5XFAD mice exhibit memory impairment in the Morris water maze task at 6 months of age [23]. In the present experiment, all mice improved in locating the submerged platform across the 8 training sessions, as shown by decreasing latency (Fig. 2a; two-way ANOVA with 4 trials/session as a repeated measure; main effect of training: $\left.F_{(7,203)}=20.01, p<0.001\right)$. Neither the main effect of treatment $\left(F_{(2,29)}=1.97, p=0.16\right)$ nor the interaction effects of treatment $\mathrm{x}$ training $\left(F_{(14,203)}=1.51, p=0.11\right)$ were significant. Post-hoc analyses revealed that performance of 5XFAD mice was poorer than those of vehicle-treated control at the session $4(p<0.05)$ and that performance of $F$. mume-treated 5XFAD mice was improved compared with vehicle-treated 5XFAD mice at the session 4 and $5(p<0.05)$ (Fig. 2a). However, no significant difference between the groups was found during the probe trials for the assessment of spatial memory retention (Fig. 2b).

Mice were then tested on object recognition task and the location novelty recognition task. Briefly, mice were initially exposed to two identical objects and re-exposed to the objects $24 \mathrm{~h}$ later when one of the objects was replaced with a novel object. After this novel object phase, the mice were tested again in a location novelty recognition test in which one of the objects was moved to a novel location in the arena. The location novelty recognition task is known to be sensitive to hippocampal damage [24]. In the novel object phase, no significant difference in the discrimination ratio was observed between the groups. However, in the new location phase, a difference in the discrimination ratio between groups was found. One-way ANOVA revealed significant between-group effects $\left(\mathrm{F}_{(2,46)}=4.360, p<0.05\right)$. Post-hoc analyses revealed that vehicle-treated 5XFAD mice spent less time exploring the object moved to novel location compared with the vehicle-treated control mice (Fig. 3a) and that the discrimination ratio in $F$. mume-treated 5XFAD mice was increased relative to vehicle-treated 5XFAD mice $(p<0.05)$ (Fig. 3a).

The final task was contextual fear conditioning, in which mice learned an association between a context (a conditioning chamber) and an aversive stimulus (a foot shock). An earlier study reported that fear memory was impaired in 5XFAD mice [19]. We measured basal levels of freezing behavior in the conditioning chamber before the foot shock delivery. Even though the basal levels of freezing behavior were low in all the groups, one-way ANOVA revealed significant between-group effects $\left(\mathrm{F}_{(2,46)}=3.08, p<0.05\right)$. Specifically, vehicle-treated 5XFAD mice exhibited slightly more freezing behavior than vehicle-treated nontransgenic mice. In contrast, the basal levels of freezing behavior were decreased in $F$. mume-treated 5XFAD mice compared with vehicle-treated 5XFAD mice. During the test session conducted $24 \mathrm{~h}$ after receiving the foot shock, the freezing levels were increased in all the groups, but with significant between-group differences $\left.\left(\mathrm{F}_{(2,}, 45\right)=7.84, p<0.01\right)$. Post-hoc analyses revealed that vehicle-treated 5XFAD mice exhibited reduced freezing behavior compared with vehicle-treated non-transgenic mice $(p<0.05)$, which implies that the fear memory was impaired in 5XFAD mice. This fear memory impairment was rescued by $F$. mume treatment $(p<0.05)$ (Fig. 3b). Together these results suggest that $F$. mume is effective in improving cognitive dysfunction in 5XFAD mice.

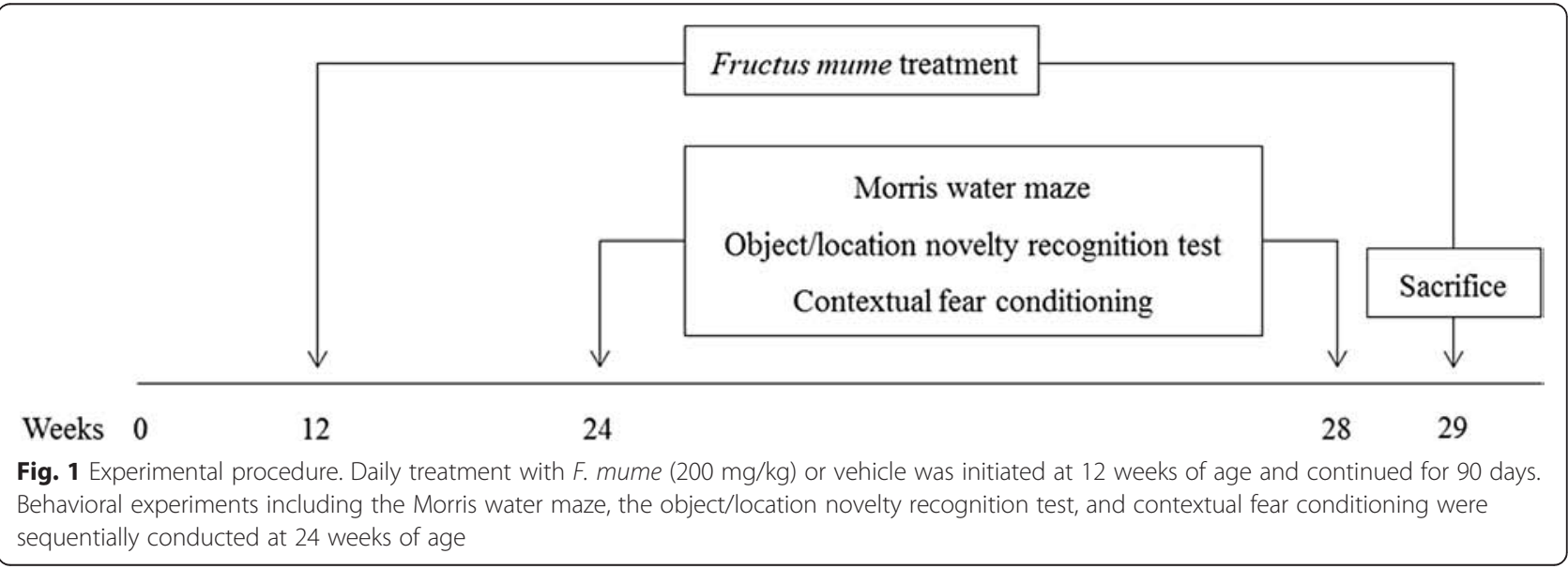



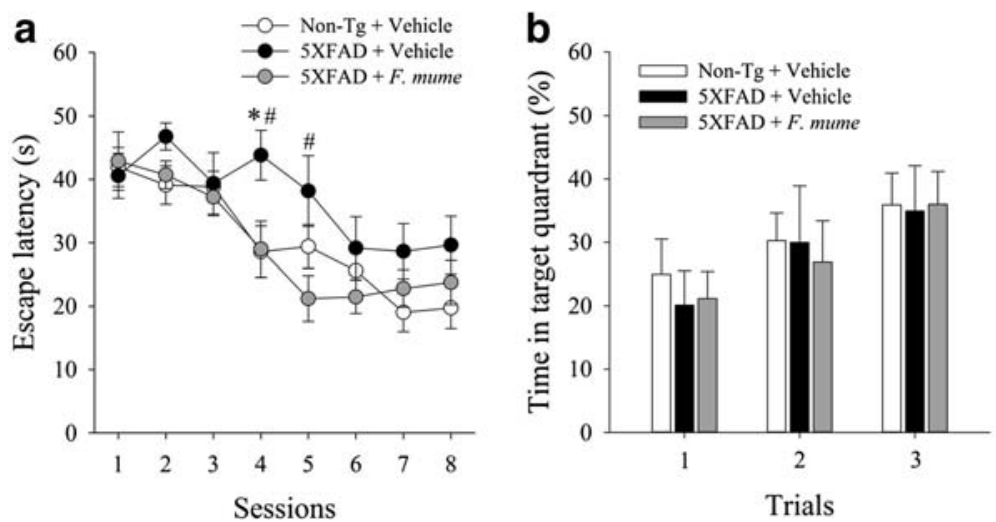

Fig. 2 Treatment with F. mume ameliorated impairments in spatial learning on the Morris water maze in 5XFAD mice. a During the hidden platform training sessions, vehicle-treated 5XFAD mice exhibited a significant learning deficit during the spatial learning training compared with vehicle-treated non-transgenic mice $\left({ }^{*} p<0.05\right)$. F. mume treatment alleviated this impairment in spatial learning $\left({ }^{*} p<0.05\right.$ versus vehicle-treated 5XFAD mice) on sessions 4 and 5. b Probe trials were conducted before the acquisition trials on sessions 5, 7, and 9 to assess the retention of spatial memory. The percentage of time spent in the target quadrant was analyzed over $30 \mathrm{~s}$. No significant differences were observed between the groups ( $n=8-14$ per group). All the data are presented as the mean \pm SEM

\section{F. mume increased levels of choline acetyltransferase expression in the hippocampus of 5XFAD mice}

It has been reported that the septo-hippocampal cholinergic system is impaired in 5XFAD mice [21, 25]. To elucidate the possible mechanisms contributing to the memory improving effect of $F$. mume, hippocampal and cortical ChAT expression levels were measured by western blot analysis (Fig. 4a). One-way ANOVA revealed significant between-group effects on the hippocampal ChAT levels $\left(\mathrm{F}_{(2,15)}=16.435, p<0.01\right)$ (Fig. 4a and b). Post-hoc analyses revealed that hippocampal ChAT levels was significantly reduced in vehicle-treated
5XFAD mice compared with vehicle-treated nontransgenic mice, which was reversed by $F$. mume treatment $(p<0.05)$ (Fig. 4a and b). No significant betweengroup differences were observed in cortical ChAT expression levels (Fig. 4a and c).

To further examine the status of cholinergic neurons in the basal forebrain of these mice, the number of ChAT-positive neurons in the medial septum (MS) and the vertical limb of the diagonal band (VBD), which provide the main cholinergic innervation to the hippocampus, were counted using immunohistochemistry (Fig. 4d). Consistent with previous studies [19], the
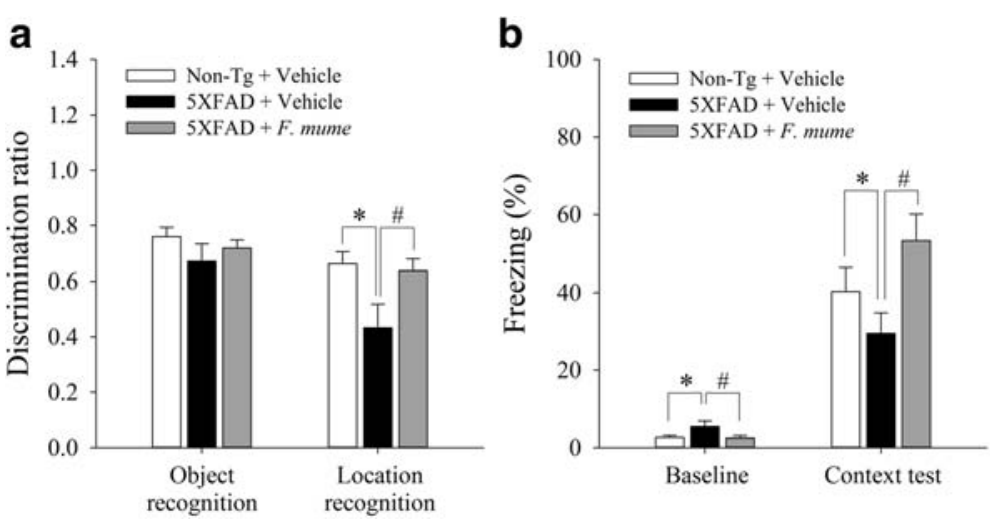

Fig. 3 F. mume treatment ameliorated impairments in recognition and fear memory in 5XFAD mice. a The object/location novelty recognition test consisted of a novel object and novel location phase. During the object phase, no significant differences in the preference for a novel object were observed between the groups, whereas, during the subsequent novel location phase, vehicle-treated 5XFAD mice exhibited less preference for the object moved to the novel location than vehicle-treated non-transgenic mice $\left.{ }^{*} p<0.05\right)$, which was reversed by $F$. mume treatment $\left({ }^{\sharp} p<0.05\right)$. b During contextual fear conditioning, mice were exposed to a context (conditioning chamber) for $148 \mathrm{~s}$ and then received three unsignaled foot shocks $(0.75$ mA, 2 s, 30 s interval). Context-associated fear memory was measured by observing freezing behavior $24 \mathrm{~h}$ later. During the test phase, vehicle-treated 5XFAD mice exhibited less freezing behavior than vehicle-treated non-transgenic mice $\left({ }^{*} p<0.05\right)$, which was reversed by treatment of F. mume $\left({ }^{\#} p<0.05\right)$. During the baseline, prior to the foot shock delivery, vehicle-treated 5XFAD mice exhibited more activity than mice in the other groups $(*, \#<0.05)(n=16-17$ per group). All the data are presented as the mean \pm SEM 

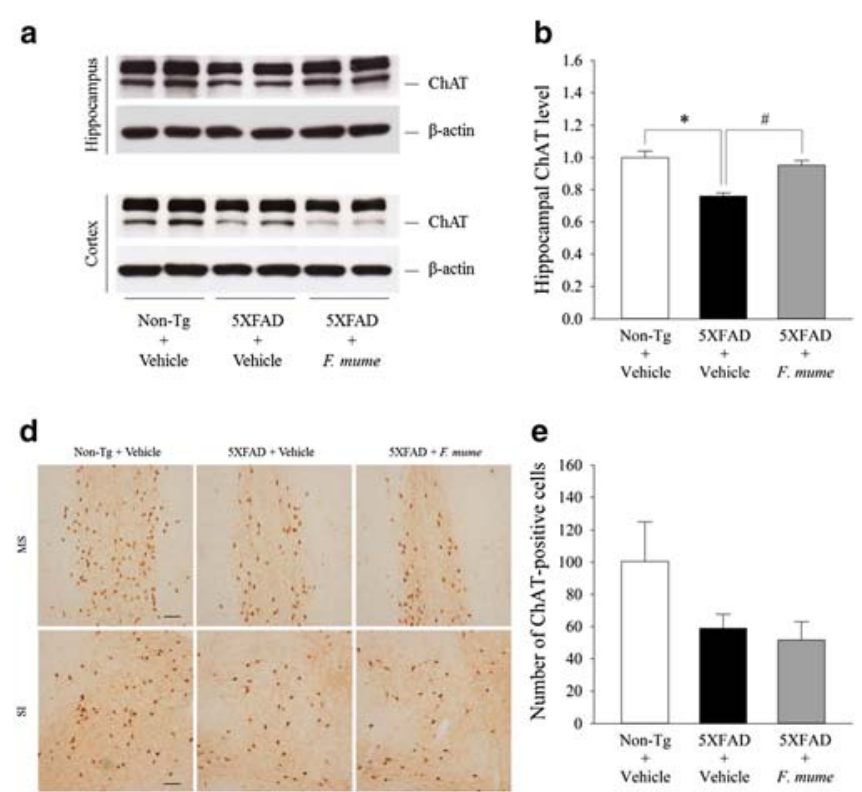
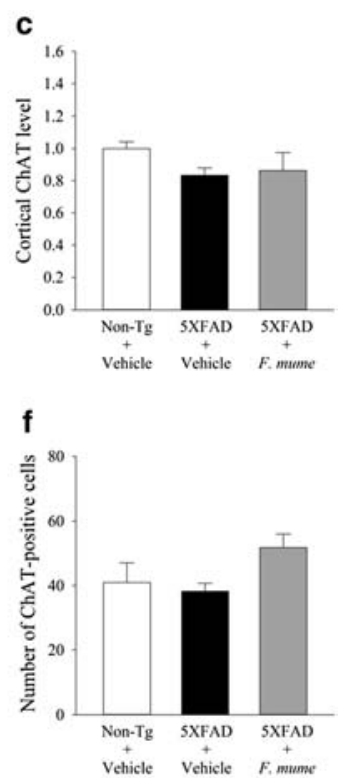

Fig. 4 F. mume treatment increased hippocampal ChAT expression in 5XFAD mice. a Hippocampal or cortical ChAT expression level was analyzed by western blot (normalized to non-transgenic + vehicle). b The expression levels of hippocampal ChAT were significantly reduced in vehicletreated 5XFAD mice compared with vehicle-treated non-transgenic mice $\left({ }^{*} p<0.05\right)$. F. mume treatment mitigated this reduction $\left({ }^{\#} p<0.05\right)(n=6$ per group). c No significant differences in expression levels of cortical ChAT were found between the groups ( $n=8-9$ per group). $\mathbf{d}$ Representative ChAT-positive neurons in the medial septum (MS) and substantia innominata (SI) are shown. Scale bar = $100 \mu$ m. e The number of ChAT-positive neurons in the MS and vertical limb of the diagonal band (VDB), which provide cholinergic innervation to the hippocampus, was counted ( $n=6$ per group). $\mathbf{f}$ The number of ChAT-positive neurons in the SI and horizontal limb of the diagonal band (HDB), which provide cholinergic innervation to the cortex, was counted ( $n=4$ per group). No significant differences were observed in the number of ChAT-positive neurons in the basal forebrain between the groups. All the data are presented as the mean \pm SEM

number of ChAT-positive neurons was reduced in the vehicle-treated 5XFAD mice and $F$. mume-treated 5XFAD mice (Fig. 4d and e). Furthermore, the number of ChAT-positive neurons that project to the cortex within the horizontal limb of the diagonal band (HDB), the magnocellular preoptic nucleus (MCPO), and the substantia innominata (SI) was not significantly different between groups (Fig. $4 \mathrm{~d}$ and $\mathrm{f}$ ). These results indicate that $F$. mume ameliorates impaired cholinergic function via the up-regulation of hippocampal ChAT levels rather than by prevention of cholinergic neurodegeneration in 5XFAD mice.

\section{Discussion}

F. mume, the smoked fruit of Prunus mume SIEB. et Zucc. (Rosaceae family), has been used to treat gastrointestinal disease, ulcer, and cough in Asian countries for thousands of years [1]. The therapeutic effects and mechanisms F. mume extract on macrophage-mediated inflammation were examined in LPS-stimulated RAW 264.7 cells. F. mume treatment inhibited the LPSinduced production of nitric oxide, prostaglandin E2, and interleukin-6 production. F. mume treatment also suppressed the signaling of mitogen-activated protein kinase and nuclear factor- $\mathrm{kB}$ activated by LPS stimulation [2]. In addition, F. mume extract was an effective treatment in an animal model of colitis [1].

Based on the results reported above, additional studies were conducted to examine the effects of F. mume extract on chronic BCCAo-induced microglial activation and the resultant cognitive improvement. F. mumetreated BCCAo rats exhibited less inflammation and more improvements in spatial memory compared with vehicle-treated BCCAo rats $[6,26]$. However, it is possible that F. mume attenuates basal forebrain cholinergic neurons impairments in rats with chronic BCCAo based on the observation of neurodegeneration in the basal forebrain cholinergic neurons of rats with chronic BCCAo [13] as well as observation that F. mume rescued scopolamine-induced spatial memory and cholinergic system impairments [7].

Hippocampal ChAT levels are markedly reduced in the brains of AD patients [27]. Studies have demonstrated that treatments leading to the up-regulation of hippocampal acetylcholine (ACh) levels reverse memory deficits [28]. For example, cognitive deficits induced by fimbria-fornix lesions are attenuated by ACh-secreting fibroblasts implanted into the hippocampus [12]. Similarly, hippocampal grafts of fetal neuronal tissue rich in cholinergic neurons reverse memory deficits produced 
by a variety of manipulations including septal, hippocampal, fimbria-fornix lesions, and selective 192 IgGsaporin-induced lesions of the septo-hippocampal ACh projection [29-31].

In addition, the expression level of acetylcholinesterase (AChE), which breaks down $\mathrm{ACh}$, has been examined in relation to $A D$ pathology. Indeed, an $A \beta$-induced AChE increase has been observed both vitro and in vivo [32-34]. There was a positive correlation between the $A \beta$ level and $A C h E$ activity in human cerebrospinal fluid [35]. AChE may interact with $A \beta$ to accelerate the deposition of amyloid plaque in the brains of $\mathrm{AD}$ patients [36]. To counteract this detrimental action of AChE in AD, AChE inhibitors such as donepezil are widely used. The therapeutic effect of AChE inhibitors has also confirmed in a mouse model of $\mathrm{AD}$ [37].

Therefore, the present study examined the efficacy of F. mume on cognitive deficits in $\mathrm{AD}$ using 5XFAD transgenic mice. Specifically, cognitive status was measured in vehicle-treated non-transgenic, vehicle-treated 5XFAD, and F. mume-treated 5XFAD mice using the Morris water maze, location novelty recognition, and contextual fear conditioning tasks. Impairments in the spatial version of the Morris water maze task, location novelty recognition task, and contextual fear conditioning task were observed in vehicle-treated 5XFAD mice relative to vehicle-treated non-transgenic mice, which is consistent with earlier reports $[20,38]$. These cognitive impairments were alleviated in the $F$. mume-treated 5XFAD mice.

Moreover, we measured hippocampal and cortical ChAT levels using western blot analysis and counted the number of cholinergic neurons in the basal forebrain using immunohistology in the vehicle-treated nontransgenic, vehicle-treated 5XFAD, and F. mume-treated 5XFAD mice, to reveal the underlying action mechanism of $F$. mume. A significant difference in the number of ChAT-positive neurons in the MS and the VBD, which innervate to the hippocampus, and the hippocampal ChAT levels was observed between vehicle-treated nontransgenic and vehicle-treated 5XFAD mice, in agreement with previous reports [21, 25]. Hippocampal ChAT levels were restored in the F. mume-treated 5XFAD mice. However, no statistical difference between vehicletreated non-transgenic and vehicle-treated 5XFAD mice was observed in the number of cortically projecting ChAT-positive neurons in the HDB, MCPO, and SI.

F. mume-treatment is reported to increase ChAT levels and decrease AChE levels in the hippocampus [7]. A study that explores AChE levels in the hippocampus and cortex of $F$. mume-treated 5XFAD mice is needed to support the hypothesis that $F$. mume acts on cholinergic neurons. In addition to improving cholinergic function, the anti-inflammatory effects of $F$. mume have been reported in a rat model of $\mathrm{VaD}$ using chronic cerebral hypoperfusion [6]. In that study, the expression levels of neuroinflammation markers such as activated microglia cells were reduced by $F$. mume. However, several studies from those in transgenic mice (including 5XFAD mice) to those in $\mathrm{AD}$ patients have demonstrated the crucial roles of neuroinflammation in $\mathrm{AD}$ pathology $[18,39,40]$. Thus, it is worth noting that the antiinflammatory effect of $F$. mume may also contribute to cognitive deficit improvements.

\section{Conclusion}

The present study demonstrated that $F$. mume improved cognitive impairments in 5XFAD mice. These results are consistent with previous reports in a chronic cerebral hypoperfusion rat model of $\mathrm{VaD}$ and in a scopolamineinduced model of memory impairment in mouse. $F$. mume also increased hippocampal ChAT expression levels, which provides evidence that $F$. mume has a therapeutic effect on impaired cholinergic functions in $\mathrm{AD}$ pathology. Therefore, these results suggest that $F$. mume may be a novel agent for the cognitive improvement and the restoration of cholinergic systems in AD.

\begin{abstract}
Abbreviations
ACh: acetylcholine; AChE: acetylcholinesterase; AD: Alzheimer's disease; APP: amyloid precursor protein; A 3 : amyloid beta; ChAT: choline acetyltransfersase; F. mume: Fructus mume; FAD: familial AD; MS: medial septum; PS1: presenilin-1; SI: substantia innominata; VDB: vertical diagonal band.
\end{abstract}

Competing interests

The authors declare that they have no competing interests.

Authors' contributions

JP carried out the behavioral tasks and immunoassays, performed the statistical analysis and drafted the manuscript. JM carried out the behavioral tasks. WJ participated in the design of the study. JH conceived of the study, and participated in its design and helped to draft the manuscript. All authors read and approved the final manuscript.

\section{Acknowledgments}

We would like to acknowledge the financial support from the R\&D Convergence Program of NST (National Research Council of Science \& Technology, G15120 and CRC-15-04-KIST) of Republic of Korea.

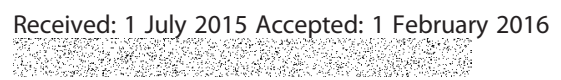

\section{References}

1. Liu L, Yuan S, Sun Y, Long Y, Li Y, Niu Y, et al. The possible mechanisms of Fructus Mume pill in the treatment of colitis induced by 2,4,6trinitrobenzene sulfonic acid in rats. J Ethnopharmacol. 2009;126(3):557-64.

2. Choi HJ, Kang OH, Park PS, Chae HS, Oh YC, Lee YS, et al. Mume Fructus water extract inhibits pro-inflammatory mediators in lipopolysaccharidestimulated macrophages. J Med Food. 2007;10(3):460-6.

3. Tomimoto $\mathrm{H}$, Ihara M, Wakita H, Ohtani R, Lin JX, Akiguchi I, et al. Chronic cerebral hypoperfusion induces white matter lesions and loss of oligodendroglia with DNA fragmentation in the rat. Acta Neuropathol. 2003; 106(6):527-34

4. Tsuchiya M, Sako K, Yura S, Yonemasu Y. Cerebral blood flow and histopathological changes following permanent bilateral carotid artery ligation in Wistar rats. Exp Brain Res. 1992;89(1):87-92. 
5. Wakita H, Tomimoto H, Akiguchi I, Kimura J. Dose-dependent, protective effect of FK506 against white matter changes in the rat brain after chronic cerebral ischemia. Brain Res. 1998;792(1):105-13.

6. Jeon WK, Ma J, Choi BR, Han SH, Jin Q, Hwang BY, et al. Effects of Fructus mume Extract on MAPK and NF-kappaB Signaling and the Resultant Improvement in the Cognitive Deficits Induced by Chronic Cerebral Hypoperfusion. Evid Based Complement Alternat Med. 2012;2012:450838.

7. Kim MS, Jeon WK, Lee KW, Park YH, Han JS. Ameliorating Effects of Ethano Extract of Fructus mume on Scopolamine-Induced Memory Impairment in Mice. Evid Based Complement Alternat Med. 2015:2015:102734.

8. Paul S, Jeon WK, Bizon JL, Han JS. Interaction of basal forebrain cholinergic neurons with the glucocorticoid system in stress regulation and cognitive impairment. Front Aging Neurosci. 2015;7:43.

9. Selkoe DJ, Schenk D. Alzheimer's disease: molecular understanding predicts amyloid-based therapeutics. Annu Rev Pharmacol Toxicol. 2003;43:545-84.

10. Park GA, Pappas BA, Murtha SM, Ally A. Enriched environment primes forebrain choline acetyltransferase activity to respond to learning experience. Neurosci Lett. 1992;143(1-2):259-62.

11. Lippa AS, Pelham RW, Beer B, Critchett DJ, Dean RL, Bartus RT. Brain cholinergic dysfunction and memory in aged rats. Neurobiol Aging. 1980; 1(1):13-9.

12. Dickinson-Anson H, Aubert I, Gage FH, Fisher LJ. Hippocampal grafts of acetylcholine-producing cells are sufficient to improve behavioural performance following a unilateral fimbria-fornix lesion. Neuroscience. 1998; 84(3):771-81.

13. Choi BR, Kwon KJ, Park SH, Jeon WK, Han SH, Kim HY, et al. Alternations of Septal-hippocampal System in the Adult Wistar Rat with Spatial Memory Impairments Induced by Chronic Cerebral Hypoperfusion. Exp Neurobiol. 2011;20(2):92-9.

14. Kumaran D, Udayabanu M, Kumar M, Aneja R, Katyal A. Involvement of angiotensin converting enzyme in cerebral hypoperfusion induced anterograde memory impairment and cholinergic dysfunction in rats. Neuroscience. 2008;155(3):626-39.

15. Contestabile A, Ciani E. The place of choline acetyltransferase activity measurement in the "cholinergic hypothesis" of neurodegenerative diseases. Neurochem Res. 2008;33(2):318-27.

16. Christensen DZ, Bayer TA, Wirths O. Intracellular Ass triggers neuron loss in the cholinergic system of the APP/PS1KI mouse model of Alzheimer's disease. Neurobiol Aging. 2010;31(7):1153-63.

17. Boncristiano S, Calhoun ME, Kelly PH, Pfeifer M, Bondolfi L, Stalder M, et al. Cholinergic changes in the APP23 transgenic mouse model of cerebral amyloidosis. J Neurosci. 2002;22(8):3234-43.

18. Oakley H, Cole SL, Logan S, Maus E, Shao P, Craft J, et al. Intraneuronal betaamyloid aggregates, neurodegeneration, and neuron loss in transgenic mice with five familial Alzheimer's disease mutations: potential factors in amyloid plaque formation. J Neurosci. 2006;26(40):10129-40.

19. Kimura $\mathrm{R}$, Ohno M. Impairments in remote memory stabilization precede hippocampal synaptic and cognitive failures in 5XFAD Alzheimer mouse model. Neurobiol Dis. 2009;33(2):229-35

20. Ohno M. Failures to reconsolidate memory in a mouse model of Alzheimer's disease. Neurobiol Learn Mem. 2009;92(3):455-9.

21. Devi L, Ohno M. Phospho-elF2alpha level is important for determining abilities of BACE1 reduction to rescue cholinergic neurodegeneration and memory defects in 5XFAD mice. PLoS One. 2010;5(9):e12974.

22. Jin Q, Lee C, Lee JW, Lee IS, Lee MK, Jeon WK, et al. Chemical Constituents from the Fruits of Prunus mume. Nat Prod Sci. 2012;18(3):200-3.

23. Kanno T, Tsuchiya A, Nishizaki T. Hyperphosphorylation of Tau at Ser396 occurs in the much earlier stage than appearance of learning and memory disorders in 5XFAD mice. Behav Brain Res. 2014;274C:302-6.

24. Save E, Poucet B, Foreman N, Buhot MC. Object exploration and reactions to spatial and nonspatial changes in hooded rats following damage to parietal cortex or hippocampal formation. Behav Neurosci. 1992;106(3):447-56.

25. Devi L, Ohno M. PERK mediates elF2alpha phosphorylation responsible for BACE1 elevation, CREB dysfunction and neurodegeneration in a mouse model of Alzheimer's disease. Neurobiol Aging. 2014;35(10):2272-81.

26. Lee KM, Bang J, Kim BY, Lee IS, Han JS, Hwang BY, et al. Fructus mume alleviates chronic cerebral hypoperfusion-induced white matter and hippocampal damage via inhibition of inflammation and downregulation of TLR4 and p38 MAPK signaling. BMC Complement Altern Med. 2015;15(1):125.

27. Ikonomovic MD, Mufson EJ, Wuu J, Cochran EJ, Bennett DA, DeKosky ST. Cholinergic plasticity in hippocampus of individuals with mild cognitive impairment: correlation with Alzheimer's neuropathology. J Alzheimers Dis. 2003;5(1):39-48.

28. Iversen SD. Behavioural evaluation of cholinergic drugs. Life Sci. 1997;60(13-14): $1145-52$.

29. Tarricone BJ, Simon JR, Li YJ, Low WC. Neural grafting of cholinergic neurons in the hippocampal formation. Behav Brain Res. 1996;74(1-2):25-44.

30. Cassel JC, Duconseille $\mathrm{E}$, Jeltsch H, Will B. The fimbria-fornix/cingular bundle pathways: a review of neurochemical and behavioural approaches using lesions and transplantation techniques. Prog Neurobiol. 1997;51(6):663-716.

31. Cassel JC, Gaurivaud M, Lazarus C, Bertrand F, Galani R, Jeltsch H. Grafts of fetal septal cells after cholinergic immunotoxic denervation of the hippocampus: a functional dissociation between dorsal and ventral implantation sites. Neuroscience. 2002;113(4):871-82.

32. Sberna G, Saez-Valero J, Li QX, Czech C, Beyreuther K, Masters CL, et al. Acetylcholinesterase is increased in the brains of transgenic mice expressing the C-terminal fragment (CT100) of the beta-amyloid protein precursor of Alzheimer's disease. J Neurochem. 1998;71(2):723-31.

33. Sberna G, Saez-Valero J, Beyreuther K, Masters CL, Small DH. The amyloid beta-protein of Alzheimer's disease increases acetylcholinesterase expression by increasing intracellular calcium in embryonal carcinoma P19 cells. J Neurochem. 1997;69(3):1177-84.

34. Hu W, Gray NW, Brimijoin S. Amyloid-beta increases acetylcholinesterase expression in neuroblastoma cells by reducing enzyme degradation. $J$ Neurochem. 2003:86(2):470-8.

35. Carroll RT, Lust MR, Emmerling MR. Beta-amyloid levels predict cholinesterase activity in human cerebrospinal fluid. Neuroreport. 1999;10(1):127-30.

36. Rees T, Hammond PI, Soreq H, Younkin S, Brimijoin S. Acetylcholinesterase promotes beta-amyloid plaques in cerebral cortex. Neurobiol Aging. 2003: 24(6):777-87.

37. Dong H, Csernansky CA, Martin MV, Bertchume A, Vallera D, Csernansky JG. Acetylcholinesterase inhibitors ameliorate behavioral deficits in the $\operatorname{Tg} 2576$ mouse model of Alzheimer's disease. Psychopharmacology (Berl). 2005; 181(1):145-52.

38. Cho WH, Park JC, Chung C, Jeon WK, Han JS. Learning strategy preference of 5XFAD transgenic mice depends on the sequence of place/spatial and cued training in the water maze task. Behav Brain Res. 2014;273:116-22.

39. Glass CK, Saijo K, Winner B, Marchetto MC, Gage FH. Mechanisms underlying inflammation in neurodegeneration. Cell. 2010;140(6):918-34.

40. Morales I, Guzman-Martinez L, Cerda-Troncoso C, Farias GA, Maccioni RB. Neuroinflammation in the pathogenesis of Alzheimer's disease. A rational framework for the search of novel therapeutic approaches. Front Cell Neurosci. 2014:8:112

\section{Submit your next manuscript to BioMed Central and we will help you at every step:}

- We accept pre-submission inquiries

- Our selector tool helps you to find the most relevant journal

- We provide round the clock customer support

- Convenient online submission

- Thorough peer review

- Inclusion in PubMed and all major indexing services

- Maximum visibility for your research

Submit your manuscript at www.biomedcentral.com/submit
Biomed Central 